\title{
Pronóstico a Largo Plazo en Niños con Crisis Epilépticas Tratadas por 5 Años
}

\author{
Dr. Marcelo Devilat B. ${ }^{1}$, Dra. Laura Germain P. 1 y \\ Dr. Bolivar Valenzuela M?

\section{Long Term Prognosis of Children with Epileptic Seizures treated for 5 Years}

\begin{abstract}
This study examines the rate of relapses in 91 children with epileptic seizures treated for a period of 5 years with antiepileptic drugs. The patients remained under observation for two years after the termination of therapy.

The results obtained (26,4\% relapses) are compared with those described in the literature. It is concluded that maintainance of treatment for periods of 3,4 or 5 years does not produce significant differences in the rate of relapses. Otherwise it was found that the great mayority of relapses appeared in the first three years after gradual reduction of drug doses.
\end{abstract}

Uno de los problemas que plantea el tratamiento antiepiléptico es su duración, es decir, cuando suspender la medicación después que un enfermo ha estado libre de crisis por un lapso determinado.

Para los adultos, los plazos de tratamiento fluctủan entre 1 a cinco años ${ }^{1-2-3-4}$. Las recomendaciones para los niños varían entre 1 a cuatro ẫos $5-6-7-8-9-10$.

La cuestión es, sin embargo, más compleja y se centra en la pregunta: ¿cuál es el riesgo de recaida que tiene un enfermo después de haber recibido tratamiento por un determinado número de años $y$ cuándo oeurien dichas recaídas?

Sorprendentemente, existe escasa investigación al respecto ${ }^{11}$, que através de experiencias programadas y controladas respondan concluyentemente a la pregunta planteadal2.

El tema adquiere, por otra parte, especial relevancia si se consideran los efectos indeseables de la medicación ${ }^{13.14}$, el efecto "Kindlig"15 y los problemas psicosociales que un prolongado tratamiento acarea a algunos enfermos ${ }^{16-17 \cdot 18-19}$.

El objetivo de la presente investigación es determinar, en un grupo de niños con crisis epilépticas tratados durante 5 años y libres crisis, el número de recaídas después de la suspensión de los medjcamentos, al cabo de un período de observación de por lo menos 2 años. Así como establecer el momento de su ocurrencia y determinar, a la luz de la literatura disponible, si un tratamiento de 5 años es más provechoso que uno más reducido. Esta. blecer además, cuál debería ser el plazo durante el cual los enfermos deben ser observados antes de darles el alta definitiva.

1 Servicio de Neurología y Psiquiatría. Hospital Luis Calvo Mackenna y División Ciencias Médicas Oriente. Facultad de Medicina. Universidad de Chile.

\section{MATERIAL Y METODO}

La muestra se compone de 91 niños con crisis epilépticas seleccionados de un grupo de 205 a los que entre 1977 y 1978 se les suspendió la medicación después de permanecer 5 años libres de crisis. Los 91 enfermos cumplían con el requisito de haber sido observados por lo menos dos años después de la suspensión total de la medicación.

El grupo estaba formado por 50 varones y 41 niñas, que habían iniciado sus crisis entre los 3 meses y los 11 años de vida.

61 enfermos (67\%) hab ian tenido 20 más crisis epilépticas afebriles antes de comenzar la ingestión del anticonvulsivante, en tanto que 26 niños (28.6\%) habian tenido súlo 1 crisis. A su vez, 4 pacientes $(4.4 \%)$ presentaron 1 crisis epiléptica ocasional (febril).

La mayoría de los enfermos, 75 (82.4\%), habían tenido crisis generalizadas (tónico-clónicas, tónicas y atónicas); un paciente eca portador de ausencias simples); 3 (3.3\%) sufrian sólo crisis parciales (simples), en tanto que 13 (14.3\%) habían presentado crisis generalizadas $y$ parciales (simples y/o complejas). No hubo niños que tuvieran Epilepsias generalizadas secundarjamente.

En 64 niños $(70.3 \%)$ no se pesquisaron antecedentes relacionables con el origen de sus crisis; 20 $(22 \%)$, tuvieron alguna patología pre-peri o postnatal. En $6(6.6 \%)$ pacientes la información fue dudosa $y$ en 1 ( $1.1 \%$ ) no habian datos al respecto.

39 enfermos $(42.8 \%)$ tenían algún familiar de primer o segundo grado portador de Epilepsia, 47 $(51.6 \%)$ no lo tenian y $5(5.5 \%)$ el antecedente se desconocia.

La mayoria de los enfermos ingirieron un sólo fármaco; $17(18.7 \%)$ recibieron 2 ó 3 anticonvulsivantes, debido a recaidas durante el tiempo en que 
fueron tratados. Los medicamentos más usados fueron fenobarbital y fenitoina, ambos en dosis de $5 \mathrm{mg}$ por kilo/día.

La edad promedio al iniciar el tratamiento fue de 3.42 años (DS \pm 2.55 ).

El tiempo de tratamiento de los niños que no recayeron inclujdo el período de reducción gradual del fármaco fue en promedio de 6.31 años (DS \pm 1.91) y el tiempo total de observación alcanzó a 9. 23 años (DS \pm 2.97 ). El grupo de enfermos que recayó durante el período de reducción gradual o después de la suspensión total del medicamento aún permanece en control.

El retiro de la medicación se hizo gradualmente a lo largo de un año (reducción gradual). En algu nos enfermos el tratamiento se prolongó por un tiempo mayor de 6 años debido a que estaban en la pubertad.

\section{RESULTADOS}

24 niños (26.4\%) recayeron, en tanto que los 67 restantes $(73.6 \%)$ no repitieron las crisis después de por lo menos 2 affos de observación sin medicamentos.

7 enfermos (29.2\%) de los 24 que presentaron recaída, la sufrieron durante el período de suspensión gradual; en 6 de ellos, la recaída se produjo dentro de los 4 primeros meses. Por otra parte, 17 pacientes $(70.8 \%$ ) repitieron sus crisis después de la supresión total del anticonvulsivante; de éstos, en $11(64.7 \%)$ la recidiva ocurrió durante el primer año de observación, en $4(23.5 \%)$ durante el segundo, en $1(5.9 \%)$ al tercer año $y$ en otro $(5.9 \%)$ al $4^{0}$ año de observación.

\section{DISCUSION}

En esta investigación, las recaídas ocurrieron en $26.4 \%$ de los casos (24/91), para un grupo de nifros con crisis convulsivas, la mayoría de ellos epilépti$\cos (61 / 91-67 \%)$.

Sorprendentemente, no existe mucha literatura (Tabla 1) que analice los resultados del tratamiento antiepiléptico en niños ${ }^{11}$ según un esquema controlado como el empleado en la presente investigación, vale decir, un grupo de niños tratados por tiempo definido, libres de crisis y observados por un lapsso mínimo determinado después de haber concluido la ingestión del anticonvulsivante.

Hollowach y Cols. ${ }^{20}$ trataron durante 4 años a 148 niños epilépticos, que estuvieron sin crisis durante todo ese tiempo: 36 (24.3\%) volvieron a tener crisis después de un periodo de observación de 5 a 12 años. Las diferencias con los resultados de la presente investigación no son significativas ( $p$ $>0.05)$ y aunque esos enfermos tenian algunas características semejantes a los nuestros, no son absolutamente comparables pues entre ellos habían 35 niños con crisis parciales o mixtas. Los
Tabla 1.

Incidencia de las recaídas después de suprimirse la medicación en niños

\begin{tabular}{|c|c|c|c|c|c|}
\hline Autores & Año & $\begin{array}{c}\text { Duración } \\
\text { terapia } \\
\text { (anfos) }\end{array}$ & $\begin{array}{c}\text { Observación } \\
\text { post-supresión } \\
\text { (años) }\end{array}$ & $\begin{array}{c}\text { Recaidas } \\
N^{0}\end{array}$ & $\%$ \\
\hline \multicolumn{6}{|l|}{ Hollowach } \\
\hline y Cols. & 1972 & 4 & $5-12$ & $36: 148$ & 24,3 \\
\hline Groh & 1975 & 3 & 3 & 152,565 & 26.9 \\
\hline $\begin{array}{l}\text { Oller-Daurclla } \\
\text { y Cols. }\end{array}$ & 1977 & +5 & +10 & $69 / 278$ & 3i) $3+$ \\
\hline Rodin y John & 1980 & 3 & 2 & $2 / 32$ & 8,2 \\
\hline Todt & 1981 & $2-4$ & 3 & $1 5 9 \longdiv { 4 3 3 }$ & 36.7 \\
\hline Hollowach & 1982 & 4 & $15-23$ & $41 / 148$ & 27,7 \\
\hline Emerson y Cols. & 1982 & 4 & $x 2.7$ & 18168 & 26.4 \\
\hline Janz y Cois. & 1982 & 2 & 2 & $25 i 59$ & 42,4 \\
\hline Devilat y Cols. & 1983 & 5 & 2 & $24 / 91$ & 26,4 \\
\hline
\end{tabular}

tadultos y niǹon

148 pacientes de la investigación anterior fueron seguidos por 10 ańos adicionales ${ }^{11}$, comprobándose 5 recardas más lo que elevó a 41 de 148 el total de recaídas para un período de observación de 15 a 23 años, to que significa un $27.7 \%$. $\mathrm{La}$ comparación con el $26.4 \%$ de la presente investigación indica que la diferencia no es significativa $(\mathrm{p}>0.05)$.

Groh $^{31}$ trató durante 3 años a 565 niños epilépticos quienes durante ese período estuvieron sin crisis. Después de 3 años de observación sin medicación habian recajdo 152 niños, vale decir, el $26.9 \%$. Nuevamente la diferencia con los resultados de la presente investigación no son significativos ( $p>0.05$ ), a pesar de que la serie de dicho autor estaba formada por 108 pacientes con crisis parciales, 58 con Síndrome de Lenox-Gastaut y 53 con Espasmos masivos y Epilepsias mixtas, lo cual naturalmente les da un carácter de menor benignidad si se la compara con nuestros enfermos, que en su mayoría tenían Epilepsias primariamen. te generalizadas del tipo Gran mal y sus variantes.

Oller-Daurella y Cols. ${ }^{2} 2$ analizaron el resultado del tratamiento antiepiléptico de 522 pacientes entre adultos y niños (pero no especifica nummero absoluto de cada uno de ellos). De ese grupo 228 enfermos fueron tratados por 5 años durante los cuales estuvieron tibres de crisis. Luego de un periodo de observación de hasta 25 años (no menor de 10), 69 pacientes habían recaido, es decir el $30.3 \%$. De nuevo, la diferencia con la presente investigación en relación a las recajdas no es significativa $(p>0.05)$. Las muestras sin embargo no son de ninguna manera absolutamente comparables dada las edades de los enfermos y la presencia de 24 enfermos con Sindrome de Lennox-Gastaut y Espasmos masivos, así como de 112 pacientes con crisis parciales.

Otros investigadores, Rodin y John ${ }^{23}$ obtuvieron un $6.2 \%$ de recaidas entre 32 niños epilépticos tratados por 3 años libres de crisis y observados durante 2 años sin medicación. Una importante 
diferencia se aprecia con los resultados obtenidos en la presente investigación, sin embargo nos parece que el reducido número de pacientes de los autores mencionados limita cualquier comparación e impide extraer conclusiones.

En un estudio hecho por Todt ${ }^{24}, 159$ niños de un grupo de $433(36.7 \%)$ recayeron después de haber sido tratados entre 2 y 4 años, lapso durante el cual estuvieron $\sin$ crisis epiléticas. El período de observación sin međicamento fue de 3 años. Este $36.7 \%$, si bien es cierto, es superior al de los autores comentados y al $26.4 \%$ de la presente investigación, la diferencia no es significativa $(p>$ 0.05 ). Por otra parte, el hecho que los enfermos de Todt hayan sido tratados por periodos diferentes de tiempo, 2 a 4 años, configuran una muestra no homogénea e incomparable con el resto de las investigaciones que se han comentado.

Por su parte, Emerson y Cols. ${ }^{25}$, trataron por 4 años libres de crisis a 68 niños epilépticos, los que fueron observados por un tiempo promedio de 2.7 años (6 meses a 6 años) sin anticonvulsivante. Durante ese lapso recayeron 18 enfermos, vale decir, el $26.4 \%$. Se observa que dicha cifra es semejante a la obtenida en la presente investigación, a pesar que los enfermos de aquellos autores tenían Epilepsias más severas que las que presentaban los niños de nuestra investigación. Además, los epilépticos de Emerson y Cols. ${ }^{25}$ tenian en aproximadamente la mitad de los casos Epilepsias parciales.

Los resultados de Janz y Cols. ${ }^{26}$ difieren de los hasta aqui analizados. En efecto, de 59 niños tratados por 2 arios sin crisis y observados por otros 2 , recayeron 25 , es decir, el $42.4 \%$. Esta cifra es significativamente superior $\left(\mathrm{X}^{2}=2.83, \mathrm{p}<0.05\right)$ a la obtenida en la presente investigación y también mas alta a la de los autores que se hán comentado aquí. Sin embargo hay que hacer notar los casos comentados son en gran parte niños portadores de Epilepsias de difícil control, Epilepsias secundariamente generalizadas o parciales, las cuales tienen mayor tendencia a recaer que las Epilepsias primariamente generalizadas ${ }^{2}$.

Er suma, cuando se tratan niños epilépticos por 3 años ${ }^{21} 4$ años $^{1-20-25}$, o por 5 años $^{22}$ (y la presente investigaciónj el porcentaje de recaídas es similar y las diferencias no son significativas. Naturalmente hay que tener presente que las muestras no son semejantes, pero la tendencia que tienen las Epilepsias infantiles a recaer independientemente de la duración del tratamiento es evidente.

Es claro también que tratamientos inferiores a 3 años en grupos de epilépticos con cuadros de difícil control, como son las Epilepsias secundariamente generalizadas y las parciales, tienden a ser menos exitosos 26

Los resultados de la presente investigación parecen sensiblemente inferiores, en términos relativos, al compararlos con los del resto de los autores que se han preocupado del tema. En efecto, las características de los niños de esta investigación son bastantes homogéneas, puesto que se trata de crisis en su mayoría generalizadas primariamente $y$ sin mucha organicidad. A su vez, los grupos de epilépticos del resto de los autores están frecuen. temente constituidos por combinaciones de Epilepsias primariamente generalizadas, parciales y secundariamente generalizadas.

Aparte de la duración total del tratamiento antiepiléptico, el modo de ređucción del fármaco es también una variable que debe ser considerada en relación a las recaídas. En general, la disminución del medicamento al final del período de tratamiento es gradual y lenta. No hay consenso acerca de cuanto tiempo debe durar este periodo, que puede ser de 1 años-7, o bien 1 a 2 años $9-10$ (Tabla 2).

\section{Tabla 2.}

Forma de suspensión de los anticonvulsivantes cn relación a las recaídas

Forma de

suspensión Recaidas Altor

\begin{tabular}{|c|c|c|c|}
\hline 2 is 3 meses & $18 / 68$ & $26 \%$ & Limerson y Cols. 1982 \\
\hline 1 an̄o & $36 / 148$ & $24 \%$ & Hollowach y Cols. 1972 \\
\hline 1 ปोั & $69 / 228$ & $30 \%$ & Oller-Daurclla 1977 \\
\hline 1 aมnัo & $24 / 91$ & $26 \%$ & Devilat y Cols. 1982 \\
\hline 2 años & $152 / 565$ & $28 \%$ & Groh 1975 \\
\hline
\end{tabular}

En esta experiencia la reducción de los anticonvulsivos fue hecha en un año y las recajdas fueron de un $26.4 \%$. Otros autores ${ }^{1-20-22}$ que tambièn en 1 año tuvieron proporciones de recaidas estadísticamente similares a las nuestras.

Por otra parte, cuando la medicación es suspendida en 2 a 3 meses $^{25}$, las recaidas no son significativamente diferentes que cuando la reducción se hace en un plazo mayor.

Si la medicación es retirada en un tiempo más largo, como 2 años 21 , tampoco se observa una significativa diferencia en relación a las recaidas como cuando se hace en 2 a 3 meses o en 1 año $20-22$.

El momento de las recaídas es un elemento de gran valor puesto que por una parte es un índice de pronóstico a largo plazo y por otra, es un indicador que permite decidir cuanto tiempo debe observarse a un enfermo después de haber suspendido la medicación (Tabla 3).

Se puede dividir el tiempo de ocurrencia de las recaídas en 3 períodos. El primero abarca el lapso durante el cual los medicamentos son retirados (en 
Tabla 3

Ticmpo de ocurrencia de las recaidas desde el inicio de la disminución del anticonvulsivante

\begin{tabular}{|c|c|c|c|c|c|c|}
\hline & \multicolumn{2}{|c|}{ Hollowach y } & \multicolumn{2}{|c|}{ Fmerson $y$} & \multicolumn{2}{|c|}{ Derilat $y$} \\
\hline & Cols. & 1972 & Cols. & 1982 & Cols. & 1982 \\
\hline $0-1$ & $22 / 148$ & $14.9 \%$ & $14 / 68$ & $20.6 \%$ & $7 / 91$ & $7.7 \%$ \\
\hline ] -2 & $4 / 148$ & $2.7 \%$ & & & $11 / 91$ & $12.1 \%$ \\
\hline $2-3$ & $4 / 148$ & $2.7 \%$ & & & $4 / 91$ & $4.4 \%$ \\
\hline 34 & & & & & $1 / 91$ & $1.1 \%$ \\
\hline $4 \ldots 5$ & $3 / 148$ & $2.0 \%$ & & & $1 / 91$ & $1.1 \%$ \\
\hline 56 & $1 / 148$ & $0.7 \%$ & & & & \\
\hline $7-8$ & $2 / 148$ & $3.3 \%$ & & & & \\
\hline & $36 / 148$ & $24.3 \%$ & $18: 68$ & $26.4 \%$ & 24,91 & $26.4 \%$ \\
\hline
\end{tabular}

general 1 año), el segundo, corresponde a $\operatorname{los} 2$ primeros años en los que el enfermo está sin anticonvulsivantes y el tercero, a partir del tercer año en que está libre medicación.

En este estudio, 7 de 91 enfermos (7.7\%) recayeron durante el primer periodo, vale decir, $7 \mathrm{de}$ los 24 que recayeron $(29.1 \%)$ lo hicieron en ese lapso. Esta cifra es muy inferior a la obtenida por Hollowach y Cols. ${ }^{20}$, quienes, también haciendo una suspensión gradual en 1 año, obtuvieron que, durante ese lapso, recayeron 22 de 148 enfermos (14.9\%) o bien, 22 de los 36 niños (61.1\%) que volvieron a tener crisis, lo hicieron dentro del primer período. las diferencias no pueden ser explicadas por ef hecho de haber suspendido la medicación durante la pubertad, que se dice representaría un especial riesgo de recaídas, puesto que a ningún niño de este grupo se le retiró la medicación durante dicho período y los enfermos de Hollowach y Cols.20, en quienes se suspendió el anticonvulsivante durante la pubertad no recayeron más frecuentemente que aquellos en los que los fátmacos fueron suspendidos antes o después de la pubertad. No tenemos explicación para interpretar las diferencias señaladas.

Por otra parte, es un hecho notable que la mayor parte de las recaidas que ocurren durante este periodo, aparecen dentro de los primeros 4 meses de iniciada la reducción gradual de las dosis del medicamento. Es así como de los 7 enfermos que volvieron a tener crisis durante el tiempo de reducción gradual, 6 lo hicieron en los 4 primeros meses de él, y uno al octavo mes. Esta situación está también en concordancia con Hollowach y Cols. ${ }^{20}$, puesto que 13 de los 22 pacientes que recayeron durante el primer período, lo hicieron dentro de los primeros 6 meses de dicho lapso.

Si el retiro de la medicación se hace en forma más rápida, 2 a 3 meses, como lo hicieron Emerson y Cols. ${ }^{25}$, lit mayoría de las recaídas ocurren dentro del primer año. Estos autores encontraron que 14 de los 68 niños $(20,6 \%)$ volvieron a tener crisis durante ese lapso, lo que representa que el $77.8 \%$ (14 de 18 ) de las recaídas aparecieron du. rante el primer año. Más aún, encontraron la mitad de ellas ocurren mientras el medicamento se está retirando ( 7 de $1838.9 \%$ ).

Se observa así que, por lo general, entre 29.1 y el $61.1 \%$ de las recaídas ocurren durante el período de suspensión gradual del fármaco y que la mayoría de ellas aparecen al comienzo de ese período. El significado de estas "crisis de retirada" no está muy claro y podrían ser no necesariamente una indicación de reiniciar un nuevo período de tratamiento ${ }^{27}$.

En el segundo período en que ocurren las re. caídas, estas aparecieron en 15 de 91 enfermos (16.5\%), es decir, 15 de los 24 pacientes que volvieron a tener crisis $(62.5 \%)$. En la serie de Hollowach y Cols., las recaídas para este periodo fueron de 8 en un total de 148 niños $(5.4 \%)$, o bien $22.2 \%$ ( 8 de 36 ) de los enfermos que volvieron a tener crisis.

Lo anterior significa que si suma el primer y el segundo periodo, se observa que a partir del inicio de la reducción gradual de lá medicación y hasta 3 años después, la mayoría de los pacientes que recaer lo hacen en ese lapso; en la presente investigaciọnt, 22 de $24(91.6 \%) y$ en ha serie de Hollowach $y$ Cols., 30 de $36(83.3 \%)$.

Si se analiza el tercer período, se observa que un pequeño nứmero de enfermos recae durante él. En efecto, de los 24 pacientes que volvieron a tener crisis, sólo 2 ( $8.3 \%$ ), recayeron. Uno lo hizo durante el tercer año y el otro, al cuarto año.

Una situación similar a la observada en la presente investigación se aprecia en la serie de Hollowach y Cols., quienes determinaron que 5 de los $36(13.8 \%)$ niños que recayeron, lo hicieron đurante este tercer período, que duró en algunos casos hasta 12 años.

Por ot ta parte, si este periodo se alarga hasta 22 años para algunos enfermos, como to hicieron esos mismos autores ${ }^{11}, 5$ nuevos pacientes recaen, elevando la cifra de 36 a 41 de un total de 148 niños epilépticos. Vale decir, el $27.7 \%$ de los pacientes que en esa muestra vuelven a tener crisis lo hacen en el tercer período cuando este se alarga hasta 22 años para algunos de ellos. Sin embargo, el índice total de recaídas para los 148 enfermos no se modifica sustancialmente, puesto que el porcentaje de recaidas sólo se eleva de 24.3 a $27.7 \%$.

Otros investigadores, Emerson y Cols. ${ }^{25}$, también han observado una situación similar a la descrita. En efecto, de los 18 enfermos, de su serie de 68, que recayerun, 3 lo hicieron el tercer periodo, es decir, el $16.7 \%$ con un tiempo máximo de observación para algunos de hasta 6 años.

En suma, los enfermos epilépticos siguen reca. yendo después de un periodo de observación de dos años sin medicación, como se demuestra en la presente investigación $y$ en la litecatura ${ }^{11-20-25}$, 
pero el número de recaídas durante ese lapso es reducido.

Por otra parte, el número de niños que vuelven a tener crisis en este tercer período no logra variar en forma significativa los indices de recaidas para el grupo total de enfermos en control.

Por lo tanto, aunque algunos autores piensan que el período de observación de los epilépticos deberia ser prolongado, pues algunos pueden recaer hasta 25 años después de haber retirado la medicación 22.28 , nosotros pensamos que un lapso de observación de 2 años sin medicamento es suficiente como para cubrir la mayoría de las recaídas. Por otra parte, cabría preguntarse si las recaidas que ocurren muy alejadas constituyen realmente una recaída o son más bien otra enfermedad epiléptica.

Por últirno es necesario mencionar que los resultados de la presente investigación y su comparación con los de la literatura son una aproximación al problema del pronóstico de la Epilepsia, puesto que no se ha mencionado aquí los factores relacionados con mal resultado final, tales como más de un tipo de crisis, crisis parciales, repetición cíclica de los ataques, comienzo muy precoz o muy tardio, inicio tardío del tratamiento, daño encefálico, alteraciones psiquiátricas, mala respuesta al medicamento y alteraciones electroencefalográf $\mathrm{i}$ $\operatorname{cas}^{29}$.

El estudio de estos factores ofrece una intere. sante perspectiva puesto que, como se ha visto, si el resultado del tratamiento es similar cuando se tratan enfermos por 3,4 ó 5 años, el análisis de las variables relacionadas con el pronóstico del cuadro, podrían servir de predictores para determinar si aquellos enfermos que no tienen factores de mal pronóstico, estarían en condiciones de recibir un tratamiento abreviado $25 \cdot 27$.

\section{RESUMEN}

Se estudió el porcentaje de recaídas en un grupo de 91 niños con crisis epilépticas, con relación a la longitud del tratamiento. Con este fin se seleccionaron 91 pacientes tratados durante 5 años con anticonvussivantes que permanecieron en observación a lo menos 2 años después de finalizado su tratamiento.

Se comparó los resultados obtenidos $(26,4 \%$ de recaídas) con los de literatura, observándose que la mantención del tratamiento por periodos de 3, 4 ó 5 años no produce diferencias significantes en los porcentajes de recaídas anotadas. Se comprobó además que la mayoría de los enfermos que recaen lo hacen dentro de los primeros 3 años a partir de la reducción gradual de los medicamentos $(91.6 \%)$.
NOTA: Además de los autores, el cuidado de los niños de esta investigación estuvo a cargo de los doctores Javier Cox, Francisco Mena, Alicia Valdivieso y Rodrigo Chamorro.

\section{REFERENCIAS}

J Penfield, W. and Jasper, H: Epilepsy and the functional anatomy of the human brain. Little, Brown and Company, Boston, 1954, pp. 565-566.

2 Merrit, H.H.: Medical treatment in epilepsy. Brit. Med. J. 1: 666, 1958.

${ }^{3}$ Lennox, W.G. and Lenwox, M.A.: Epilepsy and related disorders. Little, Brown and Company, Boston, 1960. $839-841$.

4 Janz, D.: Indikation zur Einleitung und Beendigung einer antiepileptischen Therapie bein Jugendlichen und Erwachsenen. In.: Kruse, R. (Hrse.): Epilepsic. Thieme, Stuttgart, 1971, S. 1 5-19.

5 Bamberger, $P h$. und Matthes, $A$ : Anfäle im Kindesalter. Karger, Basel, 1959, S. 345.

6 Loiseau, $P_{\text {.: }}$ Quand faut-il arreter un traitment anticpileptique? Nouv. Presse Med. 1 : $47(1), 1972$.

7 Lagos, J.C.: Seizures, epilepsy and your child. Harper and Row, 1974, pp. 104

8 Matthes, A.: Epilepsic. Diagnostik und Therapie für Klinik und Praxis. Thieme, Stuttgart, 1977, S. $150-15 \mathrm{I}$.

9 Kruse, R.: Prinzipien für die Finleitung und Becndjgung ciner Antikonvulsiven Langzcitherapic im Kindes und Jugendalter. Kicier Kongress 1979.

10 Kellermann, K.: Therapie der Epilepsie im Kindesalter. Disct. Med. Wschr, 103: 492, 1978.

11 Hollowach, J., Thurston, D.L., Hixon, B.B. and Keller, A.J.: Prognosis in childhood epilepsy. Additional follow-up of 148 children 15 to 23 years after withdrawal of anticonvulsant therapy. New Fingl. J. Med. 306: 831, 1982.

12 janz, $D$.: In tema di remisione e di ricomparsa di crisi durante e dopo il trattamento famacologico dell'epilessia. Boll. Lega It. Epil. 39: 95, 1982.

13 Reynolds, E.H.: Clronic antiepileptic toxicity. A review. Fpilepsiá 16:319-352, 1975 .

14 Stores, G.: Schoolchildren with epilepsy at risk for learning and behaviour problems. Develop. Mod. Child. Neurol. 20: $502,1978$.

I 5 Liringston, $K$ : Kindling - a new experimental model of epilepsy. In.: Jones, M.O. (Ed.): Epilepsy 79. The British Fpilepsy Association, London, 1979, pp. $55-59$.

16 Magendzo, S., Devilat, M., Astaburuaga, K, Mena, P. y Rauch, $M_{\text {: }}$ : Opinión acerca de epilepsia en tres grupos de madres y los efectos que tiene la educación. Rev. Chil. Pediat. 50: 87, 1979.

17 Ryan, R., Kempner, $K$. and Emlen, A.: The stigma of epilepsy as a sclf-concept. Epilepsia 21:433, 1980.

18 Ward, F. and Bower, B.: A study of cerrain social aspects of epilepsy in childhood. In.: Jones, M.O. (Ed.): Epilepsy 78. The British Lipilepsy Association, London, 1978 , pp. $27-34$.

19 Hoppe. R.: Die Epilepsic aus sozial medizinischer Sicht. Fortsche. Neurol. Psychiat 48: 101, 1980.

20 Hollowach, J., Thurston, D.L. and O'Leary, J.: Prognosis in childhood epilepsy. Follow-up study of 148 cases in wich therapy had been suspended after prolonged anticonvulsant control. New Engl. J. Med. 286: $169,1972$.

21 Groh, C.: Zur Frage der Heilharkeit kindlicher Lipilepsien. Wien Klin. Wochenschr. 87: 1-23, 1975. 
22 Oller-Daurella, L., Oller F-V, L. and Pamies, R.; Clinical, therapeutic and social status of epileptic patient without seizures for more than five years. In. Penry, J.K. (Ed.): Epilepsy, The Eighth International Symposium. Raven, New York, 1977, Pp. 69-75.

23 Roding, E.A. and John, G.: Withdrawal of anticonvulsant medications in successfully treated patients with epilepsy. In.: Wada, J.A. and Penry, J.K. (eds.): Advances in Epileptology: The Xth. Epilepsy International Symposium. Raven, New York, 1980, pp. $183-186$.

24 Todt, H.: Zur Spätprognose kindticher EpilepsieErgebnisse einer prospektiven Iängsschittsstudie. Dtsch. Gesundh-Wesen 36: 2012, 1981.

25 Emerson, R., D'Souza, B.J., Vining. E.P., Holden,
K.R., Mellits, E.D. and Freeman. J.M.: Stopping medication in childxen with epilepsy. Predictors of outcome. New Engl. J. Med. 304: $1125,1981$.

26 Janz, D. Kem, A., Mössinger, H.J. und Puhlmann. U.: Rückfall-Prognose nach Reduktion der Medikamente bei Epilepsiebehandlung. In.: Remschmidt. H., Rentz, R. und Jungmann, J. (Eds.): Epilepsie, 1981. (lm Verlag).

27 Gordon, $N$ : Duration of treatment for childhood epilepsy. Develop. Med. Child. Neurol. 24: 84, 1982.

28 Oller-Daurella, $L$, and Marquez, J.: Survey of 100 epileptics who have not had seizures for ten years or more. Epilepsia 13: 161, 1972.

29 Wolf, P.: Verlaufsprognose von Epilepsie. Akt. Neurol. 6: $197,1979$. 